\title{
Standardization of Face Image Sample Quality*
}

\author{
Xiufeng Gao ${ }^{1}$, Stan Z. Li ${ }^{2}$, Rong $\mathrm{Liu}^{2}$, and Peiren Zhang ${ }^{1}$ \\ ${ }^{1}$ University of Science and Technology of China \\ Hefei 230026, China \\ ${ }^{2}$ Center for Biometrics and Security Research \& \\ National Laboratory of Pattern Recognition \\ Institute of Automation, Chinese Academy of Sciences, Beijing 100080, China \\ http://www.cbsr.ia.ac.cn
}

\begin{abstract}
Performance of biometric systems is dependent on quality of acquired biometric samples. Poor sample quality is a main reason for matching errors in biometric systems and may be the main weakness of some implementations. In this paper, we present an approach for standardization of facial image quality, and develop facial symmetry based methods for its assessment by which facial asymmetries caused by non-frontal lighting and improper facial pose can be measured. Experimental results are provided to illustrate the concepts, definitions and effectiveness.
\end{abstract}

Keywords: Biometric sample quality, facial symmetry, local features, methodology, standardization.

\section{Introduction}

Sample quality has significant impact on accuracy of biometric recognition. Poor sample quality is a main reason for matching errors in biometric systems and may be the main weakness of some implementations. Automatic biometric image quality assessment may help improve system performance. It can be used to monitor image quality for different applications, capture devices, enrollment and recognition algorithms. The use of face image quality metrics to enhance the overall performance of the system is growing.

Fig. 1 shows a framework of a biometric recognition system using an image sample quality assessment component. Biometric images are preprocessed and their quality is evaluated. Only images with acceptable quality are received for recognition; others are discarded. Thus some of recognition errors can be avoided and matching time expense can be saved for a large biometric database. Also, in some security situation, quality assessment can give an alert when someone does not want to be recognized on purpose, for example, a criminal hiding himself. The quality value can be sent to recognition algorithm and helps to improve its accuracy. For example, the threshold can be decreased if an image's quality is low. In this way, low false reject rate (FRR) can be achieved.

\footnotetext{
* The original ideas presented in this paper belong to CBSR and the work was performed at CBSR.
} 


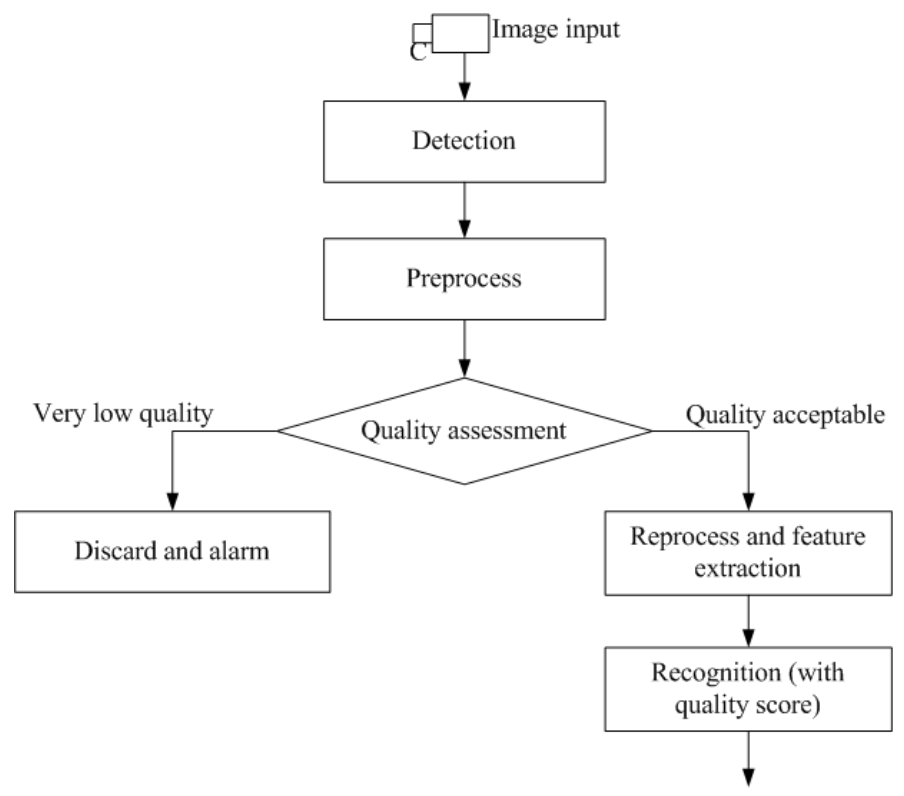

Fig. 1. A framework of biometric recognition using quality assessment

Standardization of quantitative face image quality score computation lays a basis for common interpretation of the quality scores. ISO/IEC WD 29794-1 [1] presents three approaches for calculating quantitative quality scores, namely "bottom-up", "top-down" and "combined". The presentation is based on the character, fidelity and utility concepts therein. ISO/IEC WD 29794-4 [2] emphasizes the use of annotated fingerprint corpus to standardize the score normalization. ISO-IEC WD 12 suggested the use of Quality Algorithm Identification (QAID), or Quality Percentile Rank upon standardization of a Quality Score Normalization (QSN) corpus. On the other hand, ISO/IEC standard WD 19794-5 3] are also made to regulate enrollment of gallery faces for applications in E-passport, national ID, and driver license applications, aimed to achieve approximately frontal illumination and pose.

A number of research papers on biometric sample quality assessment are published in past years. Chen [4] proposed a wavelet-based quality measure for iris image which improveed the performance. Youmaran [5] introduced a definition of biometric information and proposed an algorithm to measure the changes in biometric sample quality resulting from image degradation. Most work on face image quality is based on general, face-nonspecific image properties, such as contrast, sharpness and illumination intensity etc [6]. These methods are not aimed to evaluate face quality degradation caused by non-frontal lighting and improper facial pose.

In this paper, we present an approach for standardization of facial image quality. Aspects of defects are categorized. A two-level quality score system, defect aspect level and overall level, is suggested. Then, we develop facial symmetry based methods for the assessment of facial image quality by proposing metrics 
measuring facial asymmetries caused by non-frontal lighting and improper facial pose. Experimental results are provided to illustrate the concepts, definitions and effectiveness. The proposed methods have been incorporated into SC37 standard working draft [7].

The remainder of this paper is organized as follows: In Section 2, an approach is presented for facial image quality, in terms of 4 categories of defects, and metrics of face image quality are proposed. In Section 3, facial symmetry based and other face quality metrics are presented. In Section 4, experimental results are demonstrated.

\section{Standardization Approach}

\subsection{Categorization of Defects}

A face image obtained from a static camera, video camera, or photo scanner is usually imperfect. It may contain defects caused by poor illumination, improper face positioning and imperfection of camera. These factors can be categorized into four aspects:

(1) Defects caused by environment

- Deviation from the symmetric lighting

- Uneven lighting on the face area

- Extreme strong or weak illumination

- Cluttered background

(2) Defects caused by camera conditions

- Low resolution

- Low contrast

- High noise

- Geometric distortion

(3) Defects caused by user's face conditions

- Heavy facial wears, such as thick or dark glasses

- Exaggerated expression

- Closed eye(s)

- Heavy makeup

(4) Defects caused by user-camera positioning

- Deviation from frontal pose (yaw, tilt, in-plane rotation)

- Too far (face too small) or too near (face too big)

- Out of focus (low sharpness)

- Partial occlusion of the face

\subsection{Approach}

The performance of an automated face recognition system is affected by the amount of defect or the degree of imperfection present in the face image. The knowledge of quality can be used to invoke appropriate processing algorithms, for example, some image enhancement or normalization algorithms prior to feature extraction, appropriate thresholds or matchers based on quality.

In this paper, we adopt the following approach for face sample quality standardization: 
(1) Specifying possible defects of face biometric samples in categories. There can be several defect aspects in each category, some having been specified in Section 2.1.

(2) Defining a Face Quality Score (FQS), to be calculated by a Face Quality Assessment Algorithm (FQAA), to evaluate possible defect in each aspects.

(3) Mapping the raw FQS of each category to a normalized quality score using an annotated Quality Score Normalized Dataset (QSND) 1]. The categorynormalized FQS indicates how good the sample is with respect to the considered category of quality.

(4) Mapping all the FQSs to an overall normalized quality score using the annotated QSND. This provides an overall evaluation of how good the sample is for biometric recognition.

An FQAA takes a face sample as its input and reports the associated quality score in some aspect. Essentially, at least one FQS should be defined for each category of defects, so that a tactic procedure can be deployed to deal with the problems.

A raw FQS should be normalized in order to achieve interoperability. This may be done by a quality score normalization procedure with a QSND 1 . In QSND, biometric samples are annotated with normalized quality scores. Such annotation may be partly derived from the results of controlled performance tests, and partly by manual labeling.

\section{$3 \quad$ Face Quality Scores}

In this section, we define FQSs based on facial symmetry, which evaluates degradation caused by non-frontal lighting and head pose by making use of face specific semantics; we also define FQSs based on the inter-eye distance and image characteristics, to evaluating.other user, camera and illumination conditions.

\subsection{Facial Symmetry Based Quality Scores}

The illumination and pose variations are two main issues that cause performance degradation for most existing systems [8]. Quality degradations caused by non-frontal lighting and facial pose may be assessed using facial symmetry. Fig. 2 gives intuitive illustrations of how illumination and pose affect the facial symmetry. Based on this, we propose the following facial symmetry based FQSs.

Facial Symmetry Analysis. The symmetry may be analyzed using some local image features, e.g., the raw image pixel values, or locally-filtered pixel values. When a local filter is chosen properly, it provides a better basis for computing facial symmetry 1 . The differences between image features at the corresponding

${ }^{1}$ Care must be taken that the filters used for the corresponding locations in the left and right halves should be appropriately mirrored. 

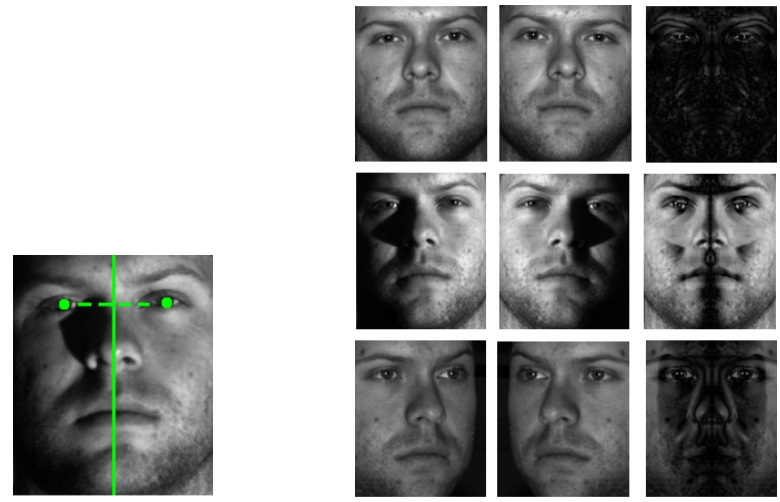

Fig. 2. Left: Division of a face into left and right half regions at the mid-line of the eyes. Right (columns 1-3): Original images, their mirror versions, and the corresponding left-right difference images.

left-right pixel locations provide local measures of asymmetry caused by nonfrontal illumination or improper head pose. If the face image is strictly left-right symmetric, the differences should all be zero.

Because the histogram of a local feature may be a more descriptive feature than the local feature itself, we further propose to use the difference between corresponding histograms of local features as a local measure of asymmetry. Consider $m$ local features produced by $m$ local filters. Assume that the histogram of a local feature consists of $n$ bins. Then the histogram at a location can be considered as a vector of $m \times n$ elements. A histogram difference between the $\ell$-th pair of histograms left-right, $H_{\ell}^{L}$ and $H_{\ell}^{R}$, can be calculated as follows

$$
D_{\ell}=\left|H_{\ell}^{L}-H_{\ell}^{R}\right|
$$

where $|\cdot|$ is some suitable form of histogram difference, e.g., city block distance (used in this paper), histogram intersection, cross-entropy, or Kullback-Leibler divergence. The larger the $D_{\ell}$ value is, the less the left-right symmetric of the face image is, and the lower the image quality is in some aspects for the subwindow at $\ell$.

Lighting and Pose Asymmetries. Lighting symmetry should be measured based on illumination sensitive image properties. For example, Local Binary Pattern (LBP) 9] code is a local feature sensitive to illumination direction, and has been used for face recognition [101112.

In this paper, we used an Adaboost learning algorithm to select the most effective subset from a large pool of LBP features 11,12, and use the local histograms of the selected LBP features as the basis for calculating facial asymmetry. A difference is derived between the corresponding left-right LBP histograms using Equ. 1. The larger the deviation, the larger the difference value, as will be seen in experiments. 
This quality score is calculated as the sum of all the histogram differences as

$$
\operatorname{Asymmetry}(I)=\sum_{\ell=1}^{L} D_{\ell}
$$

where $L$ is the number of left-right histogram pairs. The larger Asymmetry $(I)$ is, the less frontal the lighting is for the face image $I$. This is used as a metric for evaluating the defect in the aspect of lighting direction.

Pose symmetry evaluation can be done based on pose-sensitive image features. In this paper, the same local LBP histogram features as used in lighting asymmetry are used for this purpose. The histogram distance are summed up to measure the pose asymmetry in the image. The larger the sum value, the more the face is deviated from the frontal pose, and the lower the image quality is in terms of pose symmetry.

The following summarizes an FQAA procedure for calculating facial symmetry based FQSs:

(1) Preprocess the cropped face image using appropriate algorithms;

(2) Perform local feature filtering using designated filters at designated locations;

(3) Calculate the difference between corresponding local feature histogram pairs;

(4) Calculate a suitable sum of the absolute values of the differences.

\subsection{Other Face Quality Scores}

User-Camera Distance. User-camera distance is recommended to be 1.2 $2.5 \mathrm{~m}$ in a typical photo studio and $0.7-1.0 \mathrm{~m}$ in a typical photo booth (ISO/IEC 19794-5 AMD 1 [3]). The distance is inversely related the size of the face. Therefore, the inter-eye distance Dist eye $_{\text {can }}$ can used to estimate the quality score for whether the user is at a proper distance from the camera

$$
\text { Dist }_{\text {UserCam }}=D\left(\text { Dist }_{\text {eye }}, \text { Dist }_{0}\right)
$$

where Dist $_{0}$ is the average number of inter-eye pixels when the user is at the recommended distance and the image capture devise is at the recommended setting, and $D$ is some function indicating deviation of Dist $_{\text {eye }}$ from Dist $t_{0}$. Note, however, different individuals have different inter-eye distances, and therefor the use of this metric needs more consideration.

Illumination Strength. Let $H_{0}$ be the histogram of image under a standard illumination strength and $H$ is the histogram of the image being assessed. $H_{0}$ provides a reference of standard illumination strength. In the case of very dark or very bright illumination, the distribution of the gray scale values is concentrated toward the lower or higher end of the histogram $H$. A quality score could be defined to be a distance between $H_{0}$ and the measured histogram $H$. 
Contrast. Given an image $I$ of size $M$ by $N$, the image contrast may be calculated as the standard deviation of the pixel values:

$$
\text { Contrast }_{I}=\sqrt{\frac{1}{M N} \sum_{x=0}^{M-1} \sum_{y=0}^{N-1}\left[I(x, y)-\frac{1}{M N} \sum_{x=0}^{M-1} \sum_{y=0}^{N-1} I(x, y)\right]^{2}}
$$

It is suggested that the image $I(x, y)$ should not be normalized for this calculation in order to reflect the true situation.

Sharpness. The sharpness of a face image refers to the degree of clarity in both coarse and fine details in the face region. The quality value for sharpness can be calculated via image gradient

$$
\text { Shapeness }_{I}=\sum_{x=1}^{M-2} \sum_{y=1}^{N-2} G(x, y)
$$

where $G(x, y)$ is the value of gradient magnitude calculated in a neighborhood of $(x, y)$.

\section{Experiments}

The following experiments examine on the facial asymmetry caused by nonfrontal lighting and improper facial poses using the facial symmetry based methods presented in Section 3.1. Images of 10 persons are used from the Yale face database B 13 .

\subsection{Lighting Symmetry}

Fig. 3 shows examples of LBP histogram differences (Equ. 11) for 3 face images of increasing deviation of lighting from the frontal direction. The differences are calculated between 2300 random pairs of LBP histograms, shown in horizontal axis. As can be seen, the larger the deviation, the larger the difference value.

Fig. 4 shows FQS (lighting asymmetry) values (Equ. 2 for the 10 persons (in horizontal axis) under the 5 illumination conditions (in 5 curves), where the values are normalized into the range $[0,100]$. For each of the 10 persons, 5 images, taken under 5 different illumination conditions, are used. The differences between 2300 pairs of corresponding histograms are calculated, and then summed according to Equ. 2. As can be seen, the larger the deviation from frontal lighting, the larger the overall asymmetry value.

\subsection{Pose Symmetry}

Fig. 5 shows FQS (pose asymmetric) values for 4 pose categories of the 10 people, calculated in the same way as in the lighting asymmetry. The Yale face database 

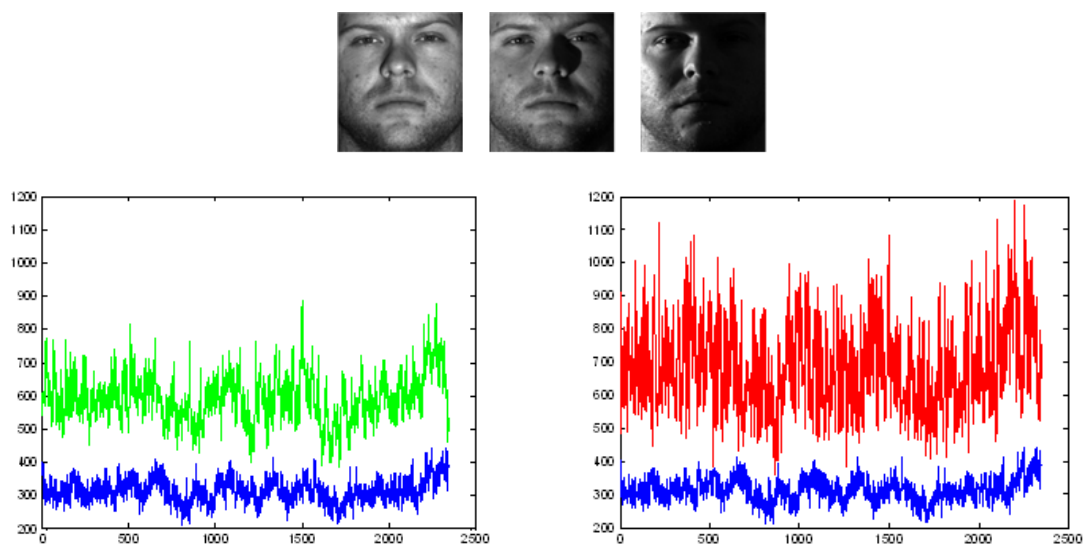

Fig. 3. Upper: 3 face images of increasing deviation of lighting from the frontal direction from left to right. Lower: histogram differences between 2300 pairs for the 1st and 2nd images (left) and for the 1st and 3rd images (right).
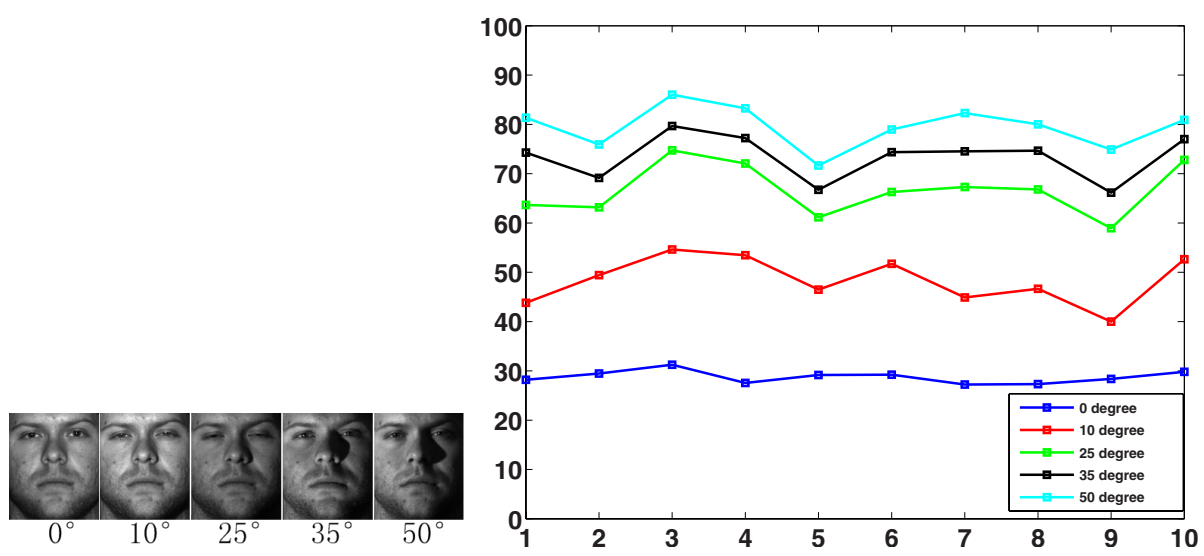

Fig. 4. Lighting FQS (lighting asymmetry) values. The 5 curves from bottom to top correspond to the face asymmetry values for the 5 light categories in the left from left to right.

B has 9 pose categories, examples being shown on the left of Fig. 5. Pose 0 is frontal (0 degree), poses $1,2,3,4$, and 5 are about 12 degrees from the camera optical axis, poses 6,7 , and 8 are about 24 degrees. Also note that poses 1, 2, $4,5,6,8$ have tilt variation. The images of poses $0,4,8,7$ are chosen for this experiment, and the corresponding FQSs are plotted on the right of the figure. As can be seen, the larger the deviation from frontal pose, the larger the overall asymmetry value. 

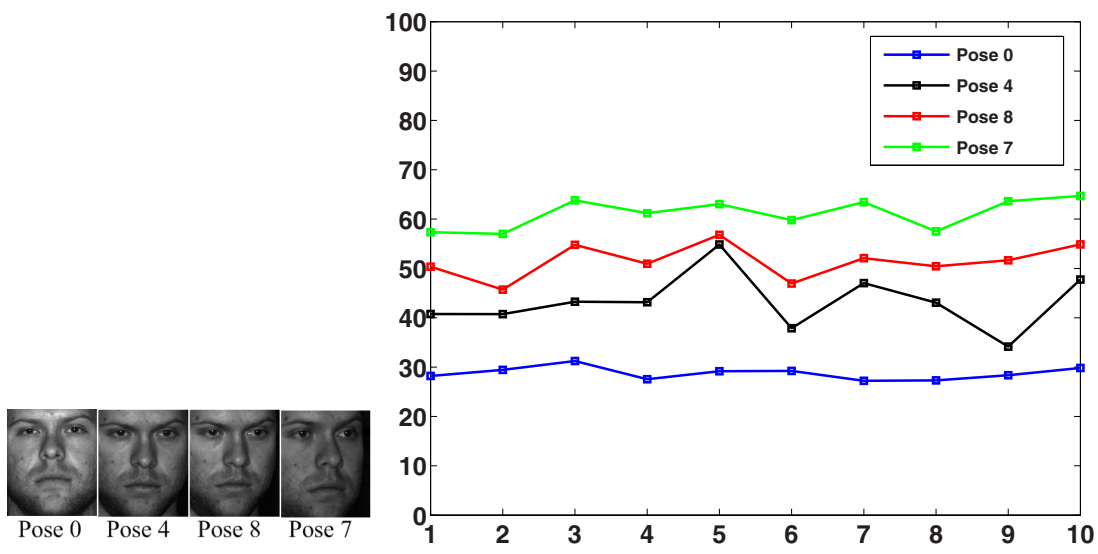

Fig. 5. Pose FQS (pose asymmetry) values. The 4 curves from bottom to top are calculated for poses $0,4,8$, and 7 .

\section{Conclusion}

We have proposed an approach for standardization of facial image quality, for which aspects of defects are categorized and a two-level quality score, aspect level and overall level, is suggested. Then, facial symmetry based methods are developed for the assessment of facial image quality for evaluation of quality degradation caused by non-frontal lighting and improper facial pose. Experimental results illustrate the concepts, definitions and effectiveness of facial symmetry based quality measures.

\section{Acknowledgements}

This work was supported by the following funding resources: National Natural Science Foundation Project \#60518002, National Science and Technology Supporting Platform Project \#2006BAK08B06, National 863 Program Projects \#2006AA01Z192 and \#2006AA01Z193, Chinese Academy of Sciences 100 people project, and the AuthenMetric Collaboration Foundation.

\section{References}

1. ISO/IEC JTC 1/SC 37 N 1477: Biometric Sample Quality Standard - Part 1: Framework (January 30, 2006)

2. ISO/IEC JTC 1/SC 37 N 1760: Biometric Sample Quality - Part 4: Fingerprint Sample Quality (August 21, 2006)

3. ISO/IEC JTC 1/SC 37 N 1511: Proposed Draft Amendment to ISO/IEC 19794-5 Face Image Data on Conditions for Taking Pictures (March 1, 2006)

4. Chen, Y., Dass, S.C., Jain, A.K.: Localized iris image quality using 2-d wavelets. In: Proc. of International Conference on Biometrics (ICB), pp. 373-381 (2006) 
5. Youmaran, R., Adler, A.: Measuring biometric sample quality in terms of biometric information. In: Biometrics Consortium Conference (2006)

6. Brauckmann, M., Werner, M.: Technical report. In: Proceddings of NIST Biometric Quality Workshop (2006), at http://www.itl.nist.gov/iad/894.03/quality/workshop/

7. ISO/IEC JTC 1/SC 37 N 1477: Biometric Sample Quality - Part 5: Face Image Data Sample Quality(Working Draft for comment) (February 12, 2007)

8. Zhao, W., Chellappa, R., Phillips, P.J., Rosenfeld, A.: Face Recognition: A Literature Survey. ACM Computing Surveys 35(4), 399-458 (2003)

9. Ojala, T., Pietikainen, M., Maenpaa, M.: Multiresolution gray-scale and rotation invariant texture classification width local binary patterns. IEEE Transactions on Pattern Analysis and Machine Intelligence 24, 971-987 (2002)

10. Ahonen, T., Hadid, A., Pietikainen, M.: Face Description with Local Binary Patterns: Application to Face Recognition. IEEE Transactions on Pattern Analysis and Machine Intelligence 28(12), 2037-2041 (2006)

11. Zhang, G., Huang, X., Li, S.Z., Wang, Y., Wu, X.: Boosting local binary pattern (lbp)-based face recognition. In: Proc. Advances in Biometric Person Authentication: 5th Chinese Conference on Biometric Recognition, pp. 179-186 (2004)

12. Li, S.Z., Chu, R., Liao, S., Zhang, L.: Illumination invariant face recognition using near-infrared images. IEEE-PAMI 26, 627-639 (2007)

13. Georghiades, A.S., Belhumeur, P.N.: From few to many: Illumination cone models for face recognition under variable lighting and pose. IEEE Transactions on Pattern Analysis and Machine Intelligence 23(6), 643-660 (2001) 